\title{
Disentangling the wind and the disk in the close surrounding of the young stellar object MWC297 with AMBER/VLTI
}

F. Malbet ${ }^{1}$, M. Benisty ${ }^{1}$, W.J. de Wit ${ }^{1}$, S. Kraus ${ }^{2}$, A. Meilland ${ }^{3}$, F. Millour $^{1,4}$, E. Tatulli ${ }^{1}$, J.-P. Berger ${ }^{1}$, O. Chesneau ${ }^{3}$, K.-H. Hofmann ${ }^{2}$, A.

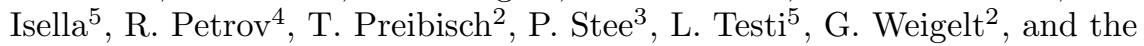
AMBER consortium

1 Laboratoire d'Astrophysique de Grenoble, UMR 5571 Université Joseph Fourier/CNRS, BP 53, F-38041 Grenoble Cedex 9, France

2 Max-Planck-Institut für Radioastronomie, Auf dem Hügel 69, D-53121 Bonn, Germany

3 Laboratoire Gemini, UMR 6203 Observatoire de la Côte d'Azur/CNRS, BP 4229, F-06304 Nice Cedex 4, France

4 Laboratoire Universitaire d'Astrophysique de Nice, UMR 6525 Université de Nice/CNRS, Parc Valrose, F-06108 Nice cedex 2, France

5 Osservatorio Astrofisico di Arcetri, Istituto Nazionale di Astrofisica, Largo E. Fermi 5, I-50125 Firenze, Italy

Summary. The young stellar object MWC 297 is a B1.5Ve star exhibiting strong hydrogen emission lines. This object has been observed by the AMBER/VLTI instrument in 2-telescope mode in a sub-region of the $\mathrm{K}$ spectral band centered around the $\mathrm{Br} \gamma$ line at $2.1656 \mu \mathrm{m}$. The object has not only been resolved in the continuum with a visibility of $0.50 \pm 0.10$, but also in the $\operatorname{Br} \gamma$ line, where the flux is about twice larger, with a visibility about twice smaller $(0.33 \pm 0.06)$. The continuum emission is consistent with the expectation of an optically thick thermal emission from dust in a circumstellar disk. The hydrogen emission can be understood by the emission of a halo above the disk surface. It can be modelled as a latitudinal-dependant wind model and it explains the width, the strength and the visibibility through the emission lines. The AMBER data associated with a high resolution ISAAC spectrum constrains the apparent size of the wind but also its kinematics.

\section{Introduction}

Pre-main sequence stars in the intermediate mass range, called Herbig Ae and Be stars (HAeBe), are observed to be surrounded by circumstellar material. It reveals itself by discrete emission lines and by continuous excess emission in the spectral energy distribution (SED). The spatial distribution of this material however has been subject to debate, where both geometrically flat disk models and spherically symmetric envelope models can reproduce the observed SED. 

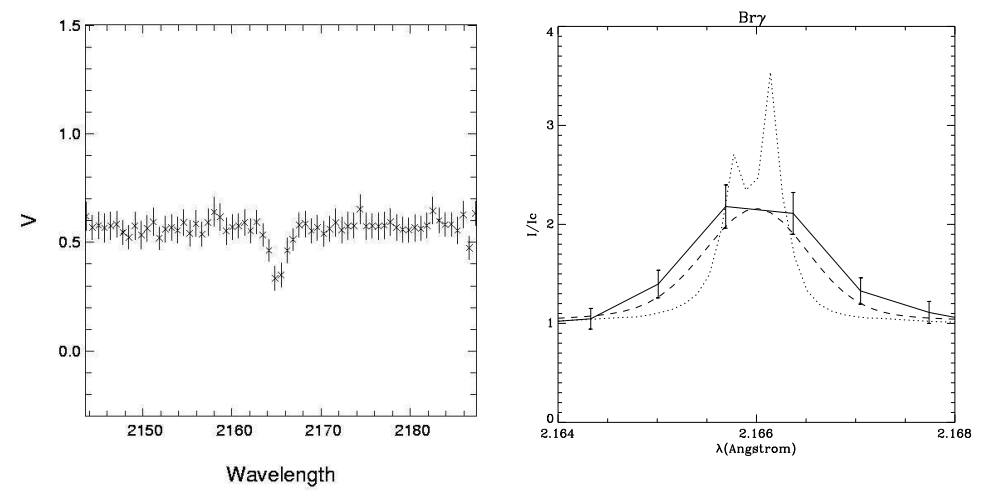

Fig. 1. Left: spectral dependence of the MWC 297 visibilities. Right: comparison of $\operatorname{Br} \gamma$ observed with AMBER (full line) and ISAAC (dotted line). The dashed line corresponds to the ISAAC spectrum convolved at the AMBER spectral resolution.

The geometry of circumstellar material near HAeBe stars seems to differ between the early-type and late-type members of the group, which is not surprising given the increasing interaction between star and disk at the earlier type stars. For the HAe stars a successful working model exist, while on the other hand, a disk structure near the HBe stars and their intricate star-disk interactions still escape a good understanding. In this study we present high spatial resolution, intermediate spectral resolution interferometric observations with AMBER of the early-type Herbig Be star MWC 297. This star displays a strong emission line spectrum corresponding to a B 1.5Ve spectral type. The rather well determined stellar parameters [1] and its high NIR luminosity render this star the perfect target to investigate in detail the geometry of the circumstellar material near the early type HAeBe stars.

\section{Observations}

MWC 297 has been observed during the second night of the first commissioning run of the AMBER instrument on the UT2-UT3 baseline of the Very Large Telescope Interferometer (VLTI). AMBER is the VLTI beam combiner in the near-infrared [2]. The instrument is based on spatial filtering with fibers and spatial beam combination along one dimension. The interferometric beam is anamorphized perpendicular to the fringe coding in order to be injected into the slit of a spectrograph. MWC 297 has been measured in the $[2100,2230 \mathrm{~nm}]$ spectral range with 1500 spectral resolution.

The results for the line visibilities are relatively consistent with all data reduction methods [3, 4], while this is not the case for the continuum visibilities. Besides the continuum visibilities in the $\mathrm{K}$ band has already been measured by other instruments like IOTA and PTI and therefore the important result is the line visibility.

Left part of Fig. 1 shows the variation of the visibility with wavelength. The continuum visibilities correspond to an average of $V_{\text {cont }}=0.50 \pm 0.10$ and the line visibility to a value of $V_{\text {line }}=0.33 \pm 0.06$. 


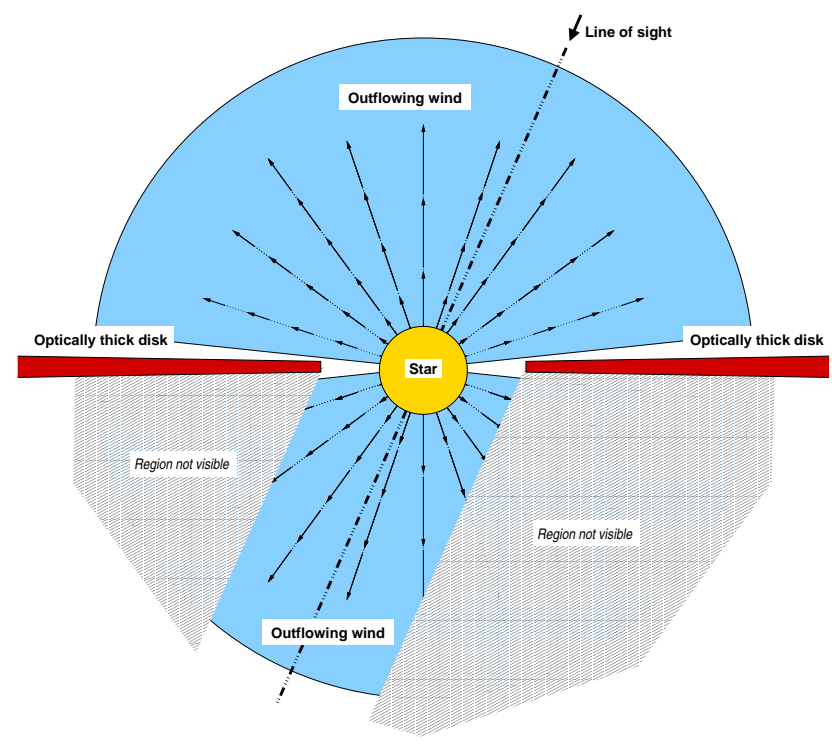

Fig. 2. Sketch of the model including an optically thick disk and an outflowing wind (edge-on view). The receding part of the wind is only partly visible because of the screen made by the optically thick disk.

MWC 297 was also observed with ISAAC at the ESO VLT UT1 telescope (see right part of Fig. $\mathbb{1}$ ) in the short wavelength medium resolution mode $(\mathcal{R}=8900)$ at the $\mathrm{Br} \gamma$ wavelength. Broad-band photometric data were collected from the litterature 1, 6, 6. Existing interferometric data for MWC 297 consist of IOTA H-band 17] and PTI K-band [8] continuum data.

\section{Modeling}

We tried to model the large body of interferometric, spectroscopic and photometric data that exists for MWC 297. The modeling is done by applying two different codes, an optically thick disk one and a stellar wind one. The disk code is designed to model the continuum radiation, whereas the stellar wind code reproduces the strong emission lines. Figure 1 represents a sketch of the combined model, where the optically thick disk and the outflowing wind are spatially independent.

\subsection{Continuum radiation: optically thick disk}

The disk model 9, 10 consists in an axisymmetric radial analytic disk structure which is heated both by stationary accretion and stellar irradiation. The disk is in hydrostatic equilibrium, non self-gravitating and the accretion flux is following the standard power law for a viscous disk. The emitted continuum flux is produced by the emission of optically thick but geometrically thin black-body radiating rings. It 

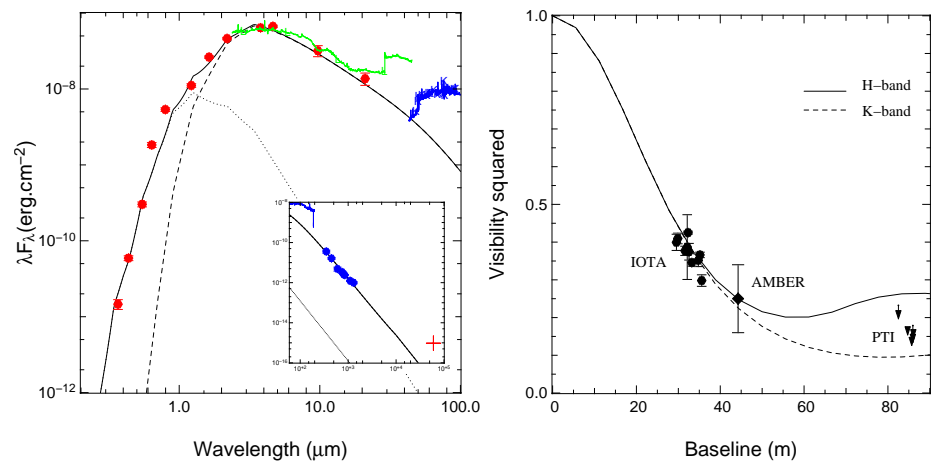

Fig. 3. Result from the optically disk model. Left panel: observed and modeled SED for MWC 297. The full dots are the continuum measurements |5], also included are the ISO SWS/LWS spectra. Dotted line is the star, dashed line the accretion disk, and the full line the resulting total flux of the model. Right panel: resulting best-fit model radial visibilities compared with AMBER, IOTA and PTI observed continuum visibilities. Full line and IOTA data are in the $H$-band, dashed line and AMBER/PTI are in the $K$-band. PTI values are upper limits.

produces an SED, and, its spatial distribution can be Fourier transformed to obtain interferometric visibilities.

We probed the sensitivities of these fits by varying the central star parameters, according to the uncertainties given by Drew et al. [1]. They derived half a spectral subtype uncertainty, and a distance error of $50 \mathrm{pc}$. The mass accretion rate is far from well determined. If the central star would be of type B2 at a distance of $200 \mathrm{pc}$, the required mass accretion rate is between 0 and $10^{-6} M_{\odot} \mathrm{yr}^{-1}$.

\subsection{Emission lines: optically thin outflowing wind}

In our model, the emission lines are produced in a circumstellar gas envelope. In order to model this line profile and the corresponding visibilities, we have used the SIMECA code 11, 12]. The solutions for all stellar latitudes are obtained by introducing a parametrized model constrained by the spectrally resolved interferometric data.

Since the SIMECA code has originally been developed to model the circumstellar environment of classical Be stars, we had to modify the code in order to interface SIMECA with the optically thick disk model described previously. We have implemented three changes:

1. The wind is no longer computed from the equator to the pole, but the computation occurs in a bipolar cone defined by a minimal angle allowing the disk to be present (see sketch in Fig. 2). We used an minimum angle of 4 degrees. The equatorial terminal velocity corresponds therefore to the terminal velocity at this minimal angle from the equatorial plane at the interface between the accretion disk and the stellar wind. 

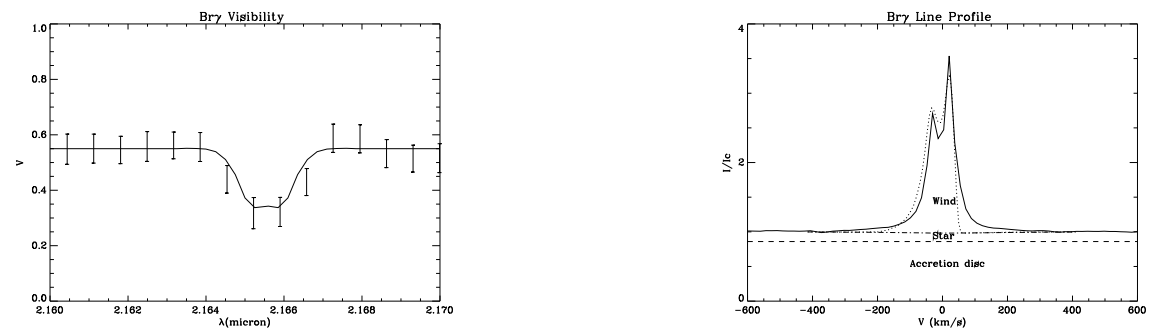

Fig. 4. Left panel: The visibility observed with AMBER (points with error bars) and the modeled outflowing wind model (full line). Right panel: double peaked Br $\gamma$ profile observed by ISAAC (full line) and modeled with the outflowing wind model (dotted line). We have also plotted the cumulative contribution of the accretion disk (dashed line) and of the star (dash-dot).

2. The disk hides the receding part of the wind. In Fig. 2, the part of the wind which is not visible from the observer is not taken into account in the outgoing flux.

3. Although the disk emission contributes less than $1 \%$ compared to the star flux in the visible (i.e. also in the $\mathrm{H} \alpha$ and $\mathrm{H} \beta$ lines) and can be neglected, at $2.1656 \mu \mathrm{m}$ the disk emission is 6.4 times larger than the stellar flux. This contribution decreases the normalized $\operatorname{Br} \gamma$ line intensity and also must be accounted for in the computation of the visibilities.

We find a successful simultaneous fit to the $\mathrm{H} \alpha, \mathrm{H} \beta$ and $\mathrm{Br} \gamma$ line profiles compatible with the observations (see Fig. 4 ) and the outflowing wind model reproduces the AMBER measured drop in visibility across the $\operatorname{Br} \gamma$ line.

We are able to reproduce quite well the shape of the $\mathrm{H} \alpha$ and $\mathrm{H} \beta$ lines and the double peaked emission of the $\operatorname{Br} \gamma$ line. The peak asymmetry of the $\operatorname{Br} \gamma$ line is also reproduced thanks to the introduction in the SIMECA code of the opacity of the disk (point 2 of SIMECA modifications). Nevertheless the agreement is not perfect in the red wing of the profile probably due to our ad-hoc way of interfacing of the wind and the disk.

\section{Discussion}

The modeling presented in the previous section, although rather successful, brings new questions on the physics of the circumstellar environment of intermediate-mass young stars.

Figure 5 shows the pole-on intensity maps of the disk model in the continuum and of the wind in the $\operatorname{Br} \gamma$ line, as well as their respective intensity profile. This is a graphical explanation of the visibilities observed by AMBER: the wind angular extension in the $\operatorname{Br} \gamma$ line is larger than the disk apparent size and therefore the visibility is smaller within the line.

Can the result obtained with these observations constrain the nature of the wind? We recall that in protoplanetary disks, two main classes of disk wind models have been proposed depending of the geometry of the magnetic field lines: the disk 

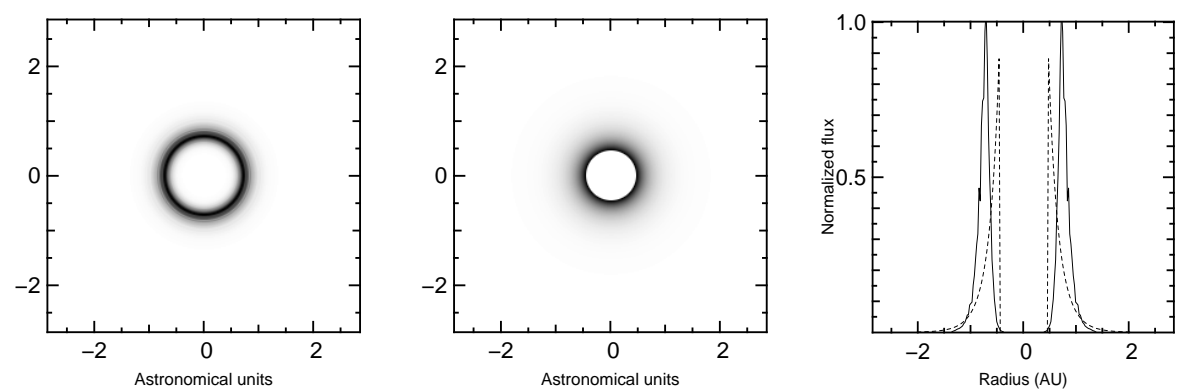

Fig. 5. Pole-on intensity maps of the wind $\operatorname{Br} \gamma$ emission (left panel) and of the $K$-band disk continuum emission (center panel). Right panel shows a radial cut of these intensity maps with the $\operatorname{Br} \gamma$ wind in solid line and the continuum disk in dashed line.

wind [13, 14] and the X-wind [15]. We cannot conclude with the present state of data since we are unable to recover a precise kinematical map of the wind. More resolution with AMBER will help to answer the question, especially using the 10000 spectral resolution mode.

Also the modeling presented in this paper does a reasonably good job in reproducing nearly all the observational data and produces fiducial physical parameters for the circumstellar environment of MWC 297. However, we derive an inclination of $\sim 20 \mathrm{deg}$ for the system, which is not consistent with a near edge-on orientation as proposed by [1]. The later is inferred from the photospheric lines that indicate a $350 \mathrm{~km} \mathrm{~s}^{-1}$ projected rotational velocity. An inclination of $20 \mathrm{deg}$ would lead to a rotation above the break-up velocity.

In conclusion, we can claim that the models of disk and wind are compatible and are probably very close from the reality. A complete and self-consistent modeling of the environment is out of the scope of the paper but would allow to better constrain the relationship between the disk and the wind at least from the observational point of view.

\section{Conclusion}

We have presented first spatially resolved observations of the disk / wind interaction in the young stellar system MWC 297 with the VLT interferometer equipped with the instrument AMBER. We have observed that the continuum visibility in the $K$ band drops from 0.50 to 0.33 in the $\mathrm{Br} \gamma$ emission line of MWC 297. The spectrum obtained with AMBER is consistent with a double peaked spectrum observed with ISAAC on the VLT, where the peaks are roughly separated by about $60 \mathrm{~km} \mathrm{~s}^{-1}$.

We have successfully modeled the circumstellar environment of MWC 297 using an optically thick geometrically thin disk and an outflowing stellar radial wind having a increasing outflowing velocity starting from the surface of the disk up to the pole. This combined model is able to reproduce many observational features like the shape of the SED over more than three decades of the wavelengths, the continuum visibilities obtained not only by AMBER but also by other infrared interferometers 
like IOTA and PTI, the spectral visibilities in the $\operatorname{Br} \gamma$ emission line as well as the $\mathrm{H} \alpha \mathrm{H} \beta$ and $\mathrm{Br} \gamma$ line profiles.

We are not yet able to constrain the exact nature of the wind and the type of connection with the disk, but we expect that future data obtained with AMBER at

a higher spectral resolution will give new kinematical information on this interesting and intriguing region.

\section{References}

1. Drew, J.E., Busfield, G., Hoare, M.G., Murdoch, K.A., Nixon, C.A., Oudmaijer, R.D.: MWC 297, B1.5Ve: a zero-age main-sequence star in the Aquila Rift. MNRAS286 (1997) 538-548

2. Petrov, R.G., et al.: Introducing the near infrared VLTI instrument AMBER to its users. Ap\&SS286 (2003) 57-67

3. Millour, F., Tatulli, E., Chelli, A., Duvert, G., Zins, G., Acke, B., Malbet, F.: Data reduction for the AMBER instrument. In: New Frontiers in Stellar Interferometry. Edited by Wesley A. Traub . Proceedings of the SPIE, Volume 5491, pp. 1222 (2004). (2004) 1222

4. Ohnaka, K., Beckmann, U., Berger, J.P., Brewer, M.K., Hofmann, K.H., Lacasse, M.G., Malanushenko, V., Millan-Gabet, R., Monnier, J.D., Pedretti, E., Schertl, D., Schloerb, F.P., Shenavrin, V.I., Traub, W.A., Weigelt, G., Yudin, B.F.: JHK'-band IOTA interferometry of the circumstellar environment of $\mathrm{R}$ CrB. A\&A408 (2003) 553-558

5. Pezzuto, S., Strafella, F., Lorenzetti, D.: On the Circumstellar Matter Distribution around Herbig Ae/Be Stars. ApJ485 (1997) 290-+

6. Mannings, V.: Submillimetre Observations of Herbig Ae/be Systems. MNRAS271 (1994) 587-+

7. Millan-Gabet, R., Schloerb, F.P., Traub, W.A.: Spatially Resolved Circumstellar Structure of Herbig AE/BE Stars in the Near-Infrared. ApJ546 (2001) 358-381

8. Eisner, J.A., Lane, B.F., Hillenbrand, L.A., Akeson, R.L., Sargent, A.I.: Resolved Inner Disks around Herbig Ae/Be Stars. ApJ613 (2004) 1049-1071

9. Malbet, F., Bertout, C.: Detecting T Tauri disks with optical long-baseline interferometry. A\&AS113 (1995) 369-+

10. Malbet, F., Lachaume, R., Berger, J.P., Colavita, M.M., Di Folco, E., Eisner, J.A., Lane, B.F., Millan-Gabet, R., Ségransan, D., Traub, W.A.: New insights on the AU-scale circumstellar structure of FU Orionis. A\&Ain press (2005)

11. Stee, P., de Araujo, F.X.: Line profiles and intensity maps from an axi-symmetric radiative wind model for Be stars. A\&A292 (1994) 221-238

12. Stee, P., de Araujo, F.X., Vakili, F., Mourard, D., Arnold, L., Bonneau, D., Morand, F., Tallon-Bosc, I.: $\gamma$ Cassiopeiae revisited by spectrally resolved interferometry. A\&A300 (1995) 219-+

13. Blandford, R.D., Payne, D.G.: Hydromagnetic flows from accretion discs and the production of radio jets. MNRAS199 (1982) 883-903

14. Casse, F., Ferreira, J.: Magnetized accretion-ejection structures. IV. Magnetically-driven jets from resistive, viscous, Keplerian discs. A\&A353 (2000) 1115-1128

15. Shu, F., Najita, J., Ostriker, E., Wilkin, F., Ruden, S., Lizano, S.: Magnetocentrifugally driven flows from young stars and disks. 1: A generalized model. ApJ429 (1994) 781-796 
F. Malbet et al. 